\title{
Response: Earlier Peak Latencies May not Fully Reflect the Robustness of Cervical Vestibular Evoked Myogenic Potential to CE-Chirp Stimulus
}

\author{
F Ceyda Akin Ocal ${ }^{1}$, Ceren Karacayli ${ }^{2}$, Volkan Kenan Coban ${ }^{1}$, and Bulent Satar ${ }^{3}$ \\ ${ }^{1}$ Department of Otorhinolaryngology, University of Health Sciences, Gulhane Traning and Research Hospital, Ankara, Turkey \\ ${ }^{2}$ Department of Audiology, University of Health Sciences, Ankara, Turkey \\ ${ }^{3}$ Department of Otorhinolaryngology, University of Health Sciences, Gulhane Medical School, Ankara, Turkey
}

\section{Dear Editor,}

We were pleased and excited that our findings in the study led to debate among scientists in the field. We would like to thank the colleagues for their interest and valuable comments on our article entitled "Can Narrow Band Chirp Stimulus Shake The Throne of $500 \mathrm{~Hz}$ Tone Burst Stimulus for Cervical Vestibular Myogenic Potentials (cVEMP)?’[1]. First of all, we have known that chirp stimulus can be used safely in auditory brainstem response (ABR) for a long time but the usage of chirp stimulus for vestibular evoked myogenic potentials (VEMPs) is a relatively new research topic. Therefore, open discussion and ongoing researches will be very useful.

It has been reported that increasing rise/fall times result in prolongation of the latencies of cVEMP [2]. Narrow-band (NB) Claus Elberling (CE) chirp is an envelope stimulus whose onset and offset can be adjusted compared to other stimuli $[3,4]$. For example, the offset of the chirp stimulus is the onset of click stimulus. It is therefore not surprising to expect early latency in chirp stimulus. We believe that the authors have misinterpreted this information.

In terms of latency, there are studies showing contradictory results in the literature $[3,5-7]$. Thus, we agree that it is necessary to be careful when interpreting the latency differences

Received September 6, 2021 / Revised September 20, 2021

Accepted September 24, 2021

\section{Address for correspondence}

F Ceyda Akin Ocal, MD

Department of Otorhinolaryngology, University of Health Sciences, Gulhane Traning and Research Hospital, Ankara 06420,Turkey

Tel+905058227471/E-mail fceydaakin@gmail.com

This is an Open Access article distributed under the terms of the Creative Commons Attribution Non-Commercial License (https://creativecommons.org/licenses/by-nc/4.0/) which permits unrestricted non-commercial use, distribution, and reproduction in any medium, provided the original work is properly cited. between different stimuli.

Walter and Cebulla [7] reported valuable findings on this subject. However, the sample size was too small $(\mathrm{n}=10)$. There are also different results in terms of latencies between the cVEMP and ocular vestibular evoked myogenic potential findings. This can make it difficult to interpret the results correctly. Wang, et al. [6] found shorter latency in chirp stimuli compared to other stimuli (click, tone pip) similar to our study. Özgür, et al. [5] studied on this subject and they found the most short latency in chirp stimuli. Interpreting these contradictory results can be difficult, as the vestibular system pathway is different from the auditory system. In case of VEMP testing, a given stimulus is to travel through some relevant auditory and vestibular structures. Irrespective of other physical characteristics of NB CE chirp stimulus, it is not a surprising finding the fact that the saccule and its fluid content stimulated by a stimulus having a wider band frequency compared to that of tonal stimulus generates stronger response on the pathway. Meanwhile, the mentioned stimuli are not physiologic stimuli for the vestibular system. Therefore, we think that more studies are needed on this subject.

Another important issue is that whether the amplitude may be a better indicator than latency. It is very difficult to confirm this with the data in the literature. Because the amplitude range is quite wide and varies among the studies [5-7]. However, while mentioning response robustness in our article, we meant waveform quality. As a result, chirp stimulus should be used more widely in the evaluation of the vestibular system in audiology clinics and these contradictions should be eliminated in the literature.

\section{Acknowledgments \\ None}




\section{Conflicts of interest}

The authors have no financial conflicts of interest.

\section{Author Contributions}

Conceptualization: all authors. Data curation: Volkan Kenan Coban. Formal analysis: F Ceyda Akin Ocal, Ceren Karacayli. Writing — original draft: F Ceyda Akin Ocal, Bulent Satar. Writing - review \& editing: Bulent Satar. Approval of final manuscript: all authors.

\section{ORCID iDs}

F Ceyda Akin Ocal

Ceren Karacayli

https://orcid.org/0000-0001-7212-2208

Volkan Kenan Coban

Bulent Satar

https://orcid.org/0000-0002-1995-0589

https://orcid.org/0000-0002-7158-8003

https://orcid.org/0000-0002-1079-2393

\section{REFERENCES}

1) Ocal FCA, Karacayli C, Coban VK, Satar B. Can narrow band chirp stimulus shake the throne of $500 \mathrm{~Hz}$ tone burst stimulus for cervical vestibular myogenic potentials? J Audiol Otol 2021;25:98-
103.

2) Cheng PW, Murofushi T. The effect of rise/fall time on vestibularevoked myogenic potential triggered by short tone bursts. Acta Otolaryngol 2001;121:696-9.

3) Zakaria MN, Zainun Z, Cheu Lih A. Considerations when analyzing vestibular evoked myogenic potential (VEMP) outcomes elicited by chirp stimulus in healthy participants. J Int Adv Otol 2015;11: 271-2.

4) Elberling C, Don M. A direct approach for the design of chirp stimuli used for the recording of auditory brainstem responses. J Acoust Soc Am 2010;128:2955-64.

5) Özgür A, Çelebi Erdivanlı Ö, Özergin Coşkun Z, Terzi S, Yiğit E, Demirci M, et al. Comparison of tone burst, click and chirp stimulation in vestibular evoked myogenic potential testing in healthy people. J Int Adv Otol 2015;11:33-5.

6) Wang BC, Liang Y, Liu XL, Zhao J, Liu YL, Li YF, et al. Comparison of chirp versus click and tone pip stimulation for cervical vestibular evoked myogenic potentials. Eur Arch Otorhinolaryngol 2014;271:3139-46.

7) Walther LE, Cebulla M. Band limited chirp stimulation in vestibular evoked myogenic potentials. Eur Arch Otorhinolaryngol 2016; 273:2983-91. 